\title{
Las universidades y el turismo: Reflexiones sobre la investigación turística en México
}

\section{Universities and tourism: Reflections on tourism research in Mexico}

\section{Alfonso de Jesús Jiménez Martínez Unidad Académica de Turismo, Universidad Autónoma de Guerrero jimenema1408@gmail.com \\ Mario Alberto Velázauez García Colegio del Estado de Hidalgo mar_002@hotmail.com}

\section{A modo de introducción. El turismo y la hipermodernidad}

En el presente escrito se busca analizar el papel de las universidades en el desarrollo Ee investigación del turismo. El objetivo es mostrar los retos que afronta un conjunto de instituciones interesadas por entender, explicar y colaborar en el desarrollo de otras actividades humanas, cuando se enfrentan a un entorno tan cambiante y complejo como el turismo. Reflexionar sobre la investigación en turismo para el caso mexicano toma relevancia si se considera el peso económico, cultural y social que tiene esta actividad para el país.

En la actualidad, el turismo constituye uno de los campos sociales donde se manifiesta de manera más clara lo que Lipovetsky denominó como la hipermodernidad; es decir, la transformación de las instituciones, prácticas e interacciones sociales orientadas hacia el consumo masivo (hiperconsumo) de lugares, mercancías y personas. El individuo libre, que se fue construyendo durante la modernidad, es sustituido por el hedonista individualizado; las personas se desplazan alrededor del mundo para satisfacer todo tipo de deseos personales y vivir experiencias "únicas” (Lipovetsky, 2006).

Vacacionar es una de las actividades que definen la nueva individualidad: soy los lugares a donde me gusta vacacionar. La importancia que ha tomado el turismo para definir la identidad descansa en dos condiciones inherentes de este hedonista individualizado: 1) cuenta con tiempo libre que le permite "disfrutar" y "explorar" nuevas realidades sociales o experiencias, y 2) tiene los recursos económicos suficientes para llevarlo a cabo. Este proceso de transformación de la construcción social de la individualidad ha sido acompañado de cambios en las instituciones: la cristalización del turismo como una práctica dentro de las sociedades contemporáneas convirtiéndose en un derecho laboral (las vacaciones pagadas por las empresas), la masificación del turismo gracias a las nuevas tecnologías de

Fecha de recepción: 10 de noviembre de 2015. Fecha de aceptación: 15 de mayo de 2016.

CÓMO CITAR: Jiménez, A. y Velázquez-García, M. A. (2017.) Las universidades y el turismo: Reflexiones sobre la investigación turística en México. Dimensiones Turísticas, 1(1), 143-155. https://doi.org/10.47557/CFZB4793 
comunicación para el transporte y, al mismo tiempo, una extensión sin precedente de las actividades y espacios considerados lugares para vacacionar. Como resultado, podemos decir que el turismo pasó de ser una actividad remota, lejana y elitista, a una práctica extendida a todos los estratos sociales (Lengkeek, 2009).

El turismo no solo se ha masificado dentro de la población, sino que también se ha expandido a diversos lugares que ahora son vistos como apropiados para esta actividad: playas, ciudades, campos, bosques e incluso las zonas donde viven los pobres. Esta expansión transforma la concepción social sobre el turismo, pero también la idea del tiempo cotidiano; el turismo puede tener lugar en el mismo espacio donde la gente vive y trabaja. Esto constituye una transición significativa si consideramos que durante los siglos xIx y xx la actividad turística normalmente estaba acotada a sitios específicos y por lo general implicaba largos viajes; el turismo ahora es parte de la cotidianidad de las grandes ciudades y zonas costeras antes dedicadas al comercio o la producción (Urry y Larsen, 2011).

Asimismo, el impacto de los procesos crecientemente automatizados en la producción de bienes y servicios tienen consecuencias como la pauperización de las condiciones laborales del mundo globalizado (Rifkin, 1996). Junto con el envejecimiento de las personas de las sociedades desarrolladas -que le dieron sustento y empuje al sistema industrial, y que se encuentran en una etapa de la vida plena de tiempo libre y con recursos, inédita para generaciones de tiempos pasados-, se cuestionan a la par las premisas de esos avances porque también existe una población desempleada, que está lejos de constituirse en demanda potencial de muchos bienes y servicios. De esa forma, en paralelo al fenómeno migratorio del Norte al Sur por ocio, se produce otro de Sur a Norte, más difícil y penoso porque la búsqueda es por trabajo y sobrevivencia que en sus territorios de origen se han visto reducidos por diversas razones (apetencias por recursos y materias primas, ideologías y religiones diferentes, guerras...).

De ese modo, además de los turistas, se observa ahora una migración de personas en procesos jubilatorios, en particular del mundo desarrollado -aunque no únicamente-, que están en la búsqueda de nuevas condiciones de clima y de economías favorables a su retiro, ubicadas frecuentemente en soleados y benévolos territorios del sur. Se vuelven así relevantes los servicios de salud y hospitalarios para los jubilados, que se hallan transformando el viaje, como un fenómeno eminentemente transitorio, para situarse en un punto intermedio entre los turistas y los inmigrantes. Esto se denomina turisgrantes, personas que se encuentran en localidades específicas durante un tiempo -más o menos largo-, ${ }^{1}$ viviendo y conviviendo como residentes locales, en el entorno natural y cultural que han elegido. Algo de eso ya se apuntaba en el capítulo de un libro de corte antropológico de finales de los años noventa (We Are Not Tourist -We Live Here, de Jacqueline Waldren, 1997).

\footnotetext{
${ }^{1}$ Un año, en la definición de la Organización Mundial del Turismo, aunque la legislación de los diferentes países puede diferir en tiempo. En México, el turista se define por una estadía de hasta seis meses, de acuerdo con la Ley de Migración que entró en vigor en 2011. De ese modo, en México, una persona que se encuentra viviendo por cinco meses 29 días será catalogada como turista en las estadísticas, aunque su papel en la comunidad sea más el de un local que vive y convive con el entorno económico, sociocultural y medioambiental del lugar donde habita.
} 


\section{A. J. Jiménez Martínez / M. A. Velázquez García}

Las transformaciones de la sociedad contemporánea y del turismo requieren un esfuerzo de comprensión integral y detallada en diferentes ámbitos (económico, político, social, ambiental y turístico), que constituye un reto formidable para las nuevas generaciones de estudiosos del turismo. La comprensión integral será muy difícil sin la investigación científica del tema turístico, y no podrá existir investigación suficiente sin los fondos para su realización. A continuación se bosquejaran algunas de las problemáticas relacionadas con la investigación del turismo en México.

\section{El turismo como objeto de estudio de la academia mexicana}

El turismo se ha convertido en uno de los temas centrales dentro de la planeación estatal mexicana. En las últimas tres décadas, esta actividad ha sido fundamental en la generación de empleo y entrada de divisas para el país. Esto contrasta con el tardío interés que ha existido por parte de la academia y el gobierno por investigar y generar datos respecto a ella. No es el objetivo de este escrito reconstruir la historia de la investigación del turismo en México - para ello sería necesario todo un libro-, sino únicamente señalar algunas tendencias y problemáticas generales.

Los primeros estudios del turismo en México, durante las décadas de los sesenta y los ochenta, se alinearon con los intereses gubernamentales y los de los empresarios del mismo sector, sobre todo en los temas vinculados con el fomento y planeación de zonas turísticas, administración de instalaciones, inversión turística, entre otros. Las primeras áreas del conocimiento que participaron en la investigación del turismo en el país fueron la economía, la administración de empresas, el urbanismo y la arquitectura, y su labor fue principalmente colaborar en hacer operativos los proyectos turísticos.

El crecimiento de los espacios, los grupos sociales y las actividades relacionadas con el turismo en México produjo un incremento de las áreas del conocimiento interesadas en analizar este campo de actividad humana. Los sociólogos, antropólogos, psicólogos, geógrafos y ambientalistas comenzaron a interesarse por estudiar los efectos que esta actividad tenía en las ciudades, las poblaciones indígenas, las zonas naturales protegidas, el acceso y uso de los recursos naturales. A diferencia de la primera etapa de la investigación en turismo, comenzaron a aparecer posturas críticas sobre los resultados del turismo como forma de generar desarrollo, las cuales provenían de las nuevas áreas del conocimiento incorporadas al análisis de estos temas así como de algunas ciencias ya involucradas desde el inicio, por ejemplo el urbanismo o la economía.

Aunque en la investigación académica fue creciendo el interés en torno al impacto social, ambiental e incluso político de esta actividad, en la base del interés gubernamental persistía el tema económico. Esto resulta fundamental, porque alrededor de esta preocupación oficial, distinta de los intereses y las cuestiones considerados importantes para las diversas disciplinas de estudio, descansan algunas problemáticas de la investigación sobre el turismo en el país. 
En México, la investigación en torno al turismo ha sido desarrollada primordialmente con el apoyo de fondos de financiamiento gubernamentales: el Consejo Nacional de Ciencia y Tecnología (Conacyt) y la Secretaría de Turismo (Sectur). En este sentido, el Estado mexicano ha constituido el principal apoyo para el desarrollo de la investigación en esta materia, sin embargo, el patrocinio gubernamental no ha sido imparcial, sino que ha devenido objeto de intereses internos que han generado una tendencia hacia temas específicos; no todos los grupos o posibles temas de investigación reciben el mismo respaldo. El gobierno mexicano tiende a favorecer aquellos relacionados con la praxis turística, es decir, la investigación que colabore con el crecimiento de los mercados, la comercialización, los precios, la competitividad y la mejor manera de incorporar productos, segmentos, y un sinfín de aspectos que caracterizan y definen el mundo actual del tema turístico.

Pareciera que los temas mencionados satisfacen todos los aspectos de interés para las sociedades receptoras en su escala nacional, regional o local, pero quedan pendientes otras cuestiones sobre el cumplimiento del objetivo último del impulso al turismo -el beneficio de la población local y/o el mejoramiento de su calidad de vida-. Aquí es necesario señalar que existe un creciente apoyo a la investigación proveniente de áreas como la sociología, la antropología, la geografía, la arquitectura o el urbanismo, las cuales realizan una crítica tanto a la misma actividad turística (sus efectos económicos, culturales y ambientales) como a la planeación y los programas gubernamentales.

Para estas reflexiones sobre la investigación en el tema turístico y el papel de las universidades, en particular de las públicas, por la dependencia y subvención de la sociedad en su conjunto, así como el papel de la Academia Mexicana de Investigación Turística (AMIT) en el tema, nos gustaría esbozar tres aspectos vinculados entre sí: la construcción social de la investigación en turismo, la responsabilidad social de la universidad y de la investigación universitaria, y la diferencia entre consultoría e investigación no contratada o libre.

\section{La construcción social de la investigación en turismo}

Como se ha tratado de mostrar, el turismo es un campo de lo social en el que pueden constatarse transformaciones fundamentales de las sociedades contemporáneas, lo cual está relacionado con los intereses económicos, sociales, culturales y de uso de los espacios que distintos grupos sociales tienen dentro de esta actividad. En el caso de la investigación es necesario preguntarnos cómo se definen los temas que la academia estudia sobre el turismo.

Una forma de responder lo anterior es mediante una propuesta sociológica: la construcción social de realidad (Ingram, Schneider y Deleon, 2007), que permite analizar los mecanismos que generan que un grupo social defina la manera de percibir su entorno social, en este caso, los temas de interés en torno al turismo en la academia mexicana. En esta perspectiva, la "realidad" (sobre el turismo en México) no es un objeto externo previamente existente, sino el resultado de una interacción entre grupos sociales con distintos 


\section{A. ). Jiménez Martínez / M. A. Velázquez García}

intereses y formas de percibir un mismo fenómeno social. Los recursos de cada grupo (capital cultural, económico, social) son determinantes en la problematización sobre el turismo. En otras palabras, es el poder de cada grupo social, más que la “objetividad” de las posturas de investigación, lo que determina la manera en que el turismo es definido dentro de las distintas universidades en México (Velázquez y Clausen, 2015).

La construcción social de las poblaciones objetivo nos permite colocar como tema central la forma en que los grupos de investigación definen los problemas, priorizan determinados temas y se conceptualizan los impactos (positivos, negativos, no deseados) que puede producir esta actividad (Ingram, Schneider y Deleon, 2007; Fox, 2001; Shaw, 2004).

En el caso del turismo, este se ha convertido en un tema central en los planes para generar desarrollo en la gran mayoría de los países de Latinoamérica. En México es una actividad estratégica. Esto descansa en una construcción gubernamental sobre el turismo como un agente central para generar crecimiento y empleo. Tal percepción del turismo ha permeado durante una buena parte del siglo xx la investigación mexicana sobre este tema: el análisis va dirigido a resolver los problemas que impiden el crecimiento de esta actividad, definir nuevas áreas de oportunidad o disminuir los efectos secundarios.

Un segundo elemento que influye en la construcción social del turismo como tema de investigación en las universidades de México es su llegada relativamente tardía, en cuanto problema social, a la institucionalización de las áreas de investigación consideradas como "centrales" para el funcionamiento de la sociedad (medicina, derecho, arquitectura) o el crecimiento (economía, ciencia política, ingenierías, etc.). Durante muchas décadas, ha existido una forma de estigmatización a los trabajos dedicados a esta materia por no ser vista como prioritaria o relacionada con el ocio. Esto correspondía a los escasos fondos que el gobierno destinó a la investigación sobre turismo durante gran parte del siglo xx.

Sin embargo, los cambios ya descritos sobre el crecimiento del turismo, en términos de los involucrados, como prestadores de servicios y la extensión territorial de su práctica, su masificación y el nuevo tipo de construcción social sobre el individuo (hedonista individualizado), pero principalmente el creciente interés de los gobiernos y las agencias internacionales de desarrollo por el turismo, han producido una transformación en la construcción social de este tema como campo para la investigación en las universidades.

El turismo se ha institucionalizado como un área de actividad social que merece ser analizada desde distintas disciplinas y genera especialistas dentro de una creciente cantidad de maestrías, doctorados y especialidades sobre turismo. Las agencias mexicanas que financian la investigación (Conacyt y organismos estatales de investigación) han comenzado a destinar importantes recursos para esta área, lo que ha originado que los grupos de investigación cuenten con mayores capitales económicos, sociales y culturales respecto a su actividad dentro del tema de turismo. 


\section{Las universidades y su responsabilidad social y de investigación}

En el marco descrito, debemos preguntarnos cuáles elementos determinan la construcción social del turismo dentro de las universidades mexicanas. El tema es relevante porque, entre otras funciones, las universidades generan conocimientos, certifican roles sociales y producen posturas críticas ante los acontecimientos sociales. En otras palabras, en sociedades como la mexicana, las universidades tienen funciones paradójicas: son agentes centrales en la reproducción del orden social, legitimando el acceso de ciertos grupos (abogados, arquitectos, médicos, etc.) al control de roles y estatus socialmente valorados, pero al mismo tiempo concentran el capital social y cultural capaz de cuestionar este mismo orden, generando modelos alternativos contra la desigualdad social.

En este mismo sentido, el lingüista y filósofo Noam Chomsky, uno de los más lúcidos y críticos personajes de la academia y la cultura universal, ha señalado algunos dilemas de la educación y de la investigación de las universidades en el contexto actual. Las universidades son - de acuerdo con Chomsky- entes cuya identidad se significa por la reflexión crítica, libre y desafiante, que coadyuva a la sociedad en la concepción que se tiene del mundo y de eso que llamamos realidad. En primer lugar, este autor expone que la educación superior en las universidades y los temas de la investigación se encuentran firmemente colocados dentro del contexto de poder que las envuelve (Chomsky, 2002). De aquí la importancia que tiene en las esferas del poder el capítulo de la tecnología para el mantenimiento del control y del mismo poder. Pero las universidades también se significan porque precisamente en sus recintos y en la investigación universitaria se han verificado muchos avances tecnológicos que definen el mundo de hoy y que han sido subvencionados por la sociedad (es decir, con dinero público). Son diversos los ejemplos que nos ofrece Chomsky, pero se incluye el armamento, la aviación, los vehículos espaciales e internet. Todos esos avances han sido financiados por gobiernos, en particular el de Estados Unidos en los últimos tiempos.

Además del poder público, en la época actual debe tenerse presente la conexión con instituciones de poder privado: las megaempresas que tienen una influencia descomunal en el mundo de las comunicaciones y el financiero, que incluye actores de la hotelería, los transportes y la comercialización global, que son también un referente de gran influencia en el mundo turístico. Chomsky nos recuerda que el sector financiero y sus dictums han dominado el orden internacional actual (2002, p. 114). Y como todos los sistemas de autoridad y dominio, "dependen crucialmente de estructuras de doctrina y creencia que les proporciona legitimación [...] ello requiere de instituciones ideológicas subordinadas y también personas para trabajar en ellas [...] lo que Antonio Gramsci llamaba 'expertos en legitimización'...” (Chomsky, 2002, p. 115). Las universidades, por su función y trascendencia, están inmersas en estos procesos de poder que tienen contradicciones de base porque, adicionalmente, son instituciones dependientes del apoyo externo para el cumplimiento de sus objetivos educativos y de investigación, esto es, para generar nuevo conocimiento, 


\section{A. J. Jiménez Martínez / M. A. Velázquez García}

y por ello no son ajenas a los sutiles métodos para asegurar la imposición de la autoridad predominante. Chomsky señala con tino el centro de flotación: las preguntas de la educación y la investigación deben tener como eje: "el conocimiento para qué y el conocimiento para quién [...] el concepto de conocimiento es defectuoso si no se acompaña de la comprensión auténtica [...] e intuición de nuestro propio lugar en el mundo material y social y la comprensión de cómo deben vivirse nuestras vidas".

De ese modo, el conocimiento debe ser una combinación de tradición científica y de ética social, porque, en última instancia, es frecuentemente el dinero público el que paga ese conocimiento. En este sentido, las universidades "deben estar libres de presiones externas y esa libertad debe ser distribuida [...] No pertenece a las administraciones ni a los profesores sino a todo el que participe en la vida universitaria -los estudiantes, los profesores y los demás empleados-" (Chomsky, 2002, p. 118), la sociedad entera se puede añadir.

En los últimos tiempos, el papel de las universidades, en particular de las públicas, se encuentra en peligro. En un artículo publicado en la revista Sociólogos (de marzo de 2014), con el revelador título "El trabajo académico, el asalto neoliberal a las universidades y cómo debería ser la educación”, Chomsky menciona la manera en la que, vistas como empresas, las universidades tienen las ataduras de la rentabilidad como principio básico de su orientación empresarial. Sin una rentabilidad adecuada, o preferentemente máxima, no se entiende su existencia. De conformidad con Chomsky, esto tiene implicaciones relevantes para la educación y para la investigación porque la libertad necesaria para la investigación científica parece ponerse en entredicho. El trabajo académico y la contratación temporal de profesores son una señal perversa de ese modelo de negocio que constituye el germen del servilismo laboral, al tener en la inseguridad laboral y la ausencia de derechos laborales y sociales, como la cobertura de salud, elementos importantes de su rentabilidad por la disminución de los costos salariales. A final de cuentas, en la educación y la investigación, el conocimiento es el principal activo que se pone en juego.

En el turismo, esos dilemas son fundamentales para la investigación porque, más que orientarse a identificar y medir los beneficios efectivos para la población local, la búsqueda de respuestas supone con frecuencia temas desde la perspectiva del turista, el mejoramiento de sus condiciones y su bienestar, que además son una importante y legítima fuente temática de la actividad académica y de investigación. Es así que temas como los recursos turísticos y el tiempo libre del que se puede disfrutar, la recreación en el destino e incluso el hedonismo y el anonimato que libera de ataduras sociales son planteados como preguntas recurrentes de la investigación académica. Y aunque esa perspectiva sea perfectamente válida, la argumentación sobre los beneficios del turismo se perpetúa hasta la náusea, sin que parezcan verificarse los beneficios de desarrollo económico y social en las comunidades locales, prometidos por el impulso de una actividad que ya parece "un cordero de Dios".

En este contexto debemos cuestionar las premisas de la argumentación que le otorga al turismo -actividad orientada por definición a lo extranjero, al que se desplaza hacia 
otros- un apoyo casi irrestricto. Asombrosamente, en los temas de la investigación turística de las universidades y de Sectur/Conacyt, cuyos fondos se han constituido en los principales recursos de la investigación turística del país y que se orientan a los procesos prácticos y de promoción e impulso a esta actividad, parecen dejarse de lado temas relevantes de la actualidad turística que pasan por las grandes empresas transnacionales y las condiciones de su operatividad en los destinos. En general, no están en discusión los efectos prometidos que llevarían al desarrollo de las comunidades locales.

\section{La diferencia principal entre consultoría e investigación universitaria}

En el contexto señalado anteriormente y en particular para el turismo, hay que diferenciar entre la investigación pública y la consultoría privada. Se ha delineado en párrafos atrás la naturaleza de la investigación universitaria (libertad temática, enfoque y método aplicable a la investigación). Se plantea como fundamental el interés del investigador sobre el tema y las crecientes aportaciones que se han efectuado desde diferentes disciplinas, de las muchas posibles en el tema turístico.

Respecto a la consultoría, es importante destacar un hecho: las preguntas de investigación las realizan las personas físicas o morales que requieren respuestas específicas y que frecuentemente están relacionadas con la incertidumbre de los nuevos negocios con cambios en las tendencias de la demanda, de la operación o de las finanzas. A cambio de ello, deben estar dispuestas a pagar por los servicios que ofrece la empresa consultora que incluye el pago del personal calificado que participa en la consultoría, así como viajes, viáticos y otros costos operativos y administrativos asociados. Las preguntas que la consultoría atiende, como se ha dicho, están normalmente vinculadas con las opciones y la conveniencia o inconveniencia de una decisión de inversión o reinversión. La entrega de las respuestas a las preguntas formuladas se establece en un tiempo determinado y los alcances de cada investigación son muy específicos. En estos casos, la naturaleza de las preguntas suele dejar fuera temas que se requieren para comprender los procesos turísticos a diferentes escalas, en toda la operación local, nacional e internacional.

El reducido universo de empresas consultoras especializadas en turismo es una particularidad de la actividad turística en México. No solo han sido pocas esas empresas, sino que parecen estar permanentemente en peligro de extinción. En otro texto hemos intentado aproximarnos a este proceso, que se vincula de manera directa con la investigación aplicada (Jiménez y Sosa, 2006). Y aquí cabe señalar una diferencia que no por obvia resulta menor tratándose de la investigación universitaria: una investigación universitaria que tiende a la consultoría es una visión que obnubila otros aspectos de una realidad que, en turismo, debería ser cada vez más cuestionada por la ausencia contundente de evidencia ante los argumentos de su promoción e impulso.

En los últimos tiempos, la orientación de la investigación fondeada con recursos públicos en México (Conacyt-Sectur) suele tener destinatario, y la convocatoria hacia los 


\section{A. J. Jiménez Martínez / M. A. Velázquez García}

investigadores normalmente se plantea con temas orientados a la praxis operativa y mercadotécnica. Aunque esos temas han auxiliado en mejorar la operatividad y la función turística, no son los únicos pertinentes. La diferencia es que estos deben ser financiados por los beneficiarios de los resultados de la investigación. Otros temas, de mayor incertidumbre en sus resultados inmediatos o de mayor escala, deberían ser financiados por las instituciones sociales encargadas de la búsqueda de nuevo conocimiento en el sector. Al final, ese debe ser el objetivo de cualquier investigación: desafiar los conocimientos establecidos y avanzar en la formulación de las teorías que buscan describir, explicar y comprender mejor nuestra realidad.

\section{Algunos temas de la investigación turística}

En México hay aspectos de la investigación turística poco explorados. Aquí se referirán solo algunos con énfasis en los aspectos relacionados con políticas públicas y los efectos económicos -en última instancia, la razón de su impulso y promoción en el nivel gubernamental-. También se intentará señalarlos en función de las diferentes escalas de observación posibles, por el tipo de información y los recursos necesarios para obtener respuestas, que podrían ser significativas en la escala internacional. Es la visión a esta escala donde prevalece la óptica derivada de la operación de las grandes operadoras turísticas internacionales (como las grandes corporaciones hoteleras, las líneas aéreas y de cruceros, y los comercializadores como las agencias on-line y los turoperadores y mayoristas), que logran una perspectiva que frecuentemente se nubla para quienes trabajan en los ámbitos local o nacional. Eso es relevante por la mirada acrítica de los organismos internacionales que, desde la década de los ochenta, están muy lejos de tener posturas inquisitivas sobre las operaciones de las empresas transnacionales (ETN) (véase, por ejemplo, los documentos del Consejo Económico y Social de las Naciones Unidas, 1975 y 1980, en los que se señalan aspectos y efectos muy interesantes de la funcionalidad turística de las ETN en ese tiempo). En la era neoliberal de los ochenta se detuvo el estudio de ese tema en el turismo.

Los temas de investigación que se enlistan a continuación solo pretenden indicar algunas de sus múltiples posibilidades dentro del contexto descrito anteriormente, relacionado con una necesaria diversidad temática.

\subsection{Escala mundial y hemisférica (hipersistema y supersistema) ${ }^{2}$}

1. Dificultades para dimensionar el efecto de los flujos en los países receptores por la omisión de un factor que incide en su valor de impacto: las estadísticas mundiales de la omт consideran solo los arribos internacionales y se omite la estadía, cuyo valor está directamente relacionado con los impactos.

${ }^{2}$ Según una terminología de la teoría general de sistemas y turismo. 
2. Efectos de las fusiones de las grandes ETN en los principales rubros de la actividad turística (aviación, hotelería y agencias).

3. Efectos del papel de los principales agentes turísticos en el control de los flujos turísticos mundiales a diferente escala.

4. Efectos de la conexión de los bancos y las instituciones financieras internacionales en la conformación de conglomerados gigantes en el sector turístico del mundo.

5. Efectos del papel de los paraísos fiscales en las tendencias observables y en la vulnerabilidad de destinos turísticos y los gobiernos ante prácticas evasoras y de lavado de dinero.

6. Efectos del papel que juegan las nuevas tecnologías en los mecanismos y tendencias de la operación turística -puntos 2 a 5 señalados-.

7. Papel de competencia hemisférica de la política fiscal en el desarrollo de los destinos y participación de las empresas turísticas (incluidas las ETN).

8. Política turística de los países de América Latina y del Caribe (escala hemisférica).

9. Política fiscal hacia el turismo en la escala hemisférica (América Latina y el Caribe).

10. Papel político de las asociaciones y consejos internacionales de carácter público, privado y social, en el impulso de medidas de promoción de políticas públicas y en el marco de las regulaciones internacionales.

\subsection{Escala nacional}

1. Revisión/discusión del dimensionamiento efectivo de los flujos de viajeros caracterizando mejor sus diferencias e indicadores concretos de su impacto. El Comité Técnico Especializado de Estadísticas Económicas del Sector Turismo (CTEEEST), constituido en el año 2009 e integrado por la Sectur, el Instituto Nacional de Estadística y Geografía, el Banco de México y el Instituto Nacional de Migración, publica las estadísticas de Datatur/Sectur con los datos más relevantes del tema. ¿No deberían integrarse miembros de la academia -neutrales en la discusión de esas estadísticas- a los trabajos de ese comité? ¿Resultan compatibles o congruentes los datos?

2. Si el objetivo último del turismo son los beneficios de la población residente, ¿son el empleo, el número de turistas y la ocupación hotelera indicadores válidos de ese cumplimiento? ¿Cómo deben medirse esos beneficios? ¿Cuáles son los indicadores pertinentes?

3. Impacto en el ingreso de las localidades y sus pobladores, según diferentes segmentos y destinos, así como caracterización detallada del empleo y sus condiciones en las distintas actividades anexas y conexas al turismo. 


\section{A. J. Jiménez Martínez / M. A. Velázquez García}

4. ¿Quién, cuánto y dónde se pagan los impuestos de las empresas turísticas, incluidas las transnacionales?

5. Papel de las nuevas tecnologías en el ingreso fiscal derivado del impulso de los flujos y las prácticas de comercialización vía internet (quién paga qué y dónde se pagan impuestos). Conexión de este tema con los CEDT (Convenios para Evitar Doble Tributación, que ha firmado México con muchos países).

6. ¿Cómo se caracteriza la práctica de devolución del IVA derivada de los consumos del turista extranjero?

7. De conformidad con la devolución del IVA, ¿qué compran los turistas: artesanía, aparatos electrónicos? ¿Cuánto se ha regresado y a quiénes? (véase análisis de Cestur-CPTM).

8. Caracterización del consumo de productos en Duty Free.

9. ¿Qué impacto tiene en la operación turística y la fiscalidad el que las empresas Duty Free de los aeropuertos sean también conglomerados internacionales?

10. Lavado de dinero y prácticas de evasión fiscal.

11. Papel político de las agrupaciones privadas (asociaciones, cámaras y consejos) y sociales (ONG y sindicatos) que funcionan como grupos de presión a diferente escala o a todas las escalas (nacional, estatal y municipal), con incidencia en el impulso de políticas públicas de turismo y en las regulaciones y normatividad de escala nacional.

12. El turismo y la gastronomía:

13. Caracterización del consumo de bebidas alcohólicas en destinos turísticos (¿cerveza, tequila?); el papel de las grandes fusiones y adquisiciones de productores por las ETN, y de los productos/marcas (Martínez-Gándara, 2008; Expansión, 2014). ¿Qué efecto tiene el hecho de que productos tradicionalmente considerados mexicanos estén bajo el control de grandes empresas transnacionales de licores ${ }^{3}$ El tema de la exclusividad (restaurantes y otros) de las dos grandes empresas (duopolio) cerveceras en México, cuya venta en locales es de una o de otra pero no simultánea. Esas prácticas, ¿limitan el desarrollo de cervezas artesanales?

14. Caracterización del uso/consumo de picantes en destinos turísticos y como identificación singular de la gastronomía mexicana (¿moles?, ¿salsas picantes? ¿Por qué si la comida de México se identifica con picantes (chile), es la Salsa Tabasco la que se ofrece en el mundo como salsa picante?

${ }^{3}$ Es el caso de cierta cerveza (aunque la cebada sea europea, hay cebada de buena calidad en México) Heineken-Cervecería Cuauhtémoc y Anheuser-Busch InBev-Grupo Modelo- (cf. Martínez-Gándara, 2005). Algo similar sucede con el tequila (véase Hernández López, 2013; Expansión, 2014; Informador.mx, 2014). 


\subsection{Escala local}

1. Beneficio concreto para la comunidad local: ¿qué y cómo se mide?

2. Empleo/recursos humanos:

- Papel del empleo incierto y basado en propinas.

- Papel de las outsourcing (pagadoras) en el desempeño de los empleados de la hotelería.

- Prácticas racistas en algunas cadenas hoteleras. El caso de una cadena hotelera española donde un alto ejecutivo selecciona al personal por su fenotipo (imagen occidental) y vetan a los trabajadores con imagen típica de nuestra gente (mexicas, mayas).

3. Ingresos gubernamentales de nivel municipal:

a) ¿Cuánto aportan las empresas al fisco municipal en México?

b) ¿Cuánto y dónde se pagan los impuestos en el caso de:4

I. aerolíneas;

II. cruceros;

III. hoteles locales/nacionales y los de cadena nacional/internacional;

IV. Agencias on-line.

4. Aportaciones al fisco municipal derivadas de las concesiones de la Zona Federal Marítimo-Terrestre.

5. Segundas residencias y dimensionamiento de la función de renta de esas viviendas.

6. Agencias mayoristas y turoperadores (nacionales e internacionales) y su influencia en la comercialización de localidades específicas.

7. Lavado de dinero y prácticas de evasión fiscal; el endeudamiento de los municipios turísticos ¿se justifica?

\section{Reflexiones finales}

En México, la investigación en turismo se ha convertido en un tema y una responsabilidad central tanto para la academia como para el gobierno y el sector privado vinculado con el desarrollo de esta actividad. Aunque cada uno de estos grupos tiene intereses diferenciados respecto al desarrollo del turismo, todos pueden compartir el interés por que el turismo se convierta en una actividad sostenible en términos económicos, sociales y culturales.

La academia tiene un conjunto de funciones y responsabilidades que la convierten en un vigilante y crítico de las actividades que ella misma avala y construye. Esto no tiene que

${ }^{4}$ Tema derivado de los convenios para evitar la doble tributación. Véase: http://www.sat.gob.mx/ informacion_fiscal/normatividad/Paginas/tratados_fiscales.aspx 


\section{A. J. Jiménez Martínez / M. A. Velázquez García}

ser una carga paradójica, sino que debe alentar para repensar sus formas de colaboración con los otros actores interesados en el turismo. Por ejemplo, convirtiéndose en un centro de encuentro y diálogo entre actores que por lo general no son considerados en los procesos de planeación y desarrollo de los programas y planes para el desarrollo del turismo.

Sin menospreciar los temas culturales, políticos o sociales relacionados con el turismo, en este trabajo hemos querido proponer una agenda de investigación del turismo cargada principalmente hacia los temas económicos. Con ello no queremos decir que la economía sea la estructura que sostiene o explica todo el funcionamiento del turismo, pero es un elemento axial que intersecta el desarrollo en este campo de acción social. El turismo es mucho más que una transacción económica, pero no deja de serlo, y este anclaje en lo económico condiciona la agencia de los actores relacionados con él.

\section{Referencias}

Chomsky, N. (2002). La responsabilidad social de la universidad. En J. E. García-Albea, N. Catalá y J. A. Díez (coords.), Los límites de la globalización. Barcelona, España: Ariel Filosofía.

Chomsky, N. (13 de marzo de 2014). El trabajo académico, el asalto neoliberal a las universidad y cómo debería ser la educación. Sociólogos.

Consejo Económico y Social de las Naciones Unidas. (1975). The impact of international tourism on the economic development of the developing countries. PRO/ECOSOC/1758 (LIV).

Consejo Económico y Social de las Naciones Unidas. (1980). Las empresas transnacionales en el turismo internacional. Doc. en español. E/C 10/68/8 de abril de 1980.

Hernández López, J. de J. (2013). Paisaje y creación de valor. La transformación de los paisajes culturales del agave y el tequila. Ciudad de México, México: El Colegio de Michoacán/Fideicomiso Felipe Teixidor y Montserrat Alfau de Texidor.

Informador.mx. (22 de noviembre de 2014). CRT ve un tequila todavía mexicano. Informador. $m x$. Recuperado de http://www.informador.com.mx/economia/2014/561104/6/ crt-ve-un-tequila-todavia-mexicano.htm

Jiménez M., A. de J. y Sosa F., A. P. (diciembre, 2006). Notas sobre las etapas de la investigación del turismo en México. Análisis del Turismo, 1, 25-32.

Lengkeek, J. (2009). Tourism studies in Belgium and the Netherlands. En G. Dann y G. Lieberman (eds.), The Sociology of Tourism: European Origins and Developments (Tourism Social Science Series, No. 12, pp. 275-297).

Lipovetsky, G. (2006). Los tiempos hipermodernos. Barcelona, España: Anagrama.

Martínez-Gándara, A. (julio-diciembre, 2008). Tequila, mezcal y cerveza: de México para el mundo. Agricultura, Sociedad y Desarrollo, 5(2), 143-150.

Rifkin, J. (1996). El fin del trabajo. Nueva tecnologías contra puestos de trabajo. El nacimiento de una nueva era. Ciudad de México, México: Paidós. 
Urry, J. y Larsen, J. (2011). The Tourist Gaze 3.0. Londres, Inglaterra: Sage.

Velázquez, M. y Clausen, H. (2015). Propuesta para el estudio de las políticas públicas del turismo: la construcción social de realidad. Topofilia. Revista de Arquitectura, Urbanismo y Territorios, V(5), 764-776.

Waldren, J. (1997). We Are Not Tourist - We Live Here. En S. Abram, J. Waldren y D. V. L. Macleod (eds.), Tourist and Tourism. Identifying with People and Places (pp. 51-70). Oxford, Inglaterra: Berg. 